A. Bayo-Salas, J. Beerten, J. Rimez, and D. Van Hertem, "Analysis of control interactions between parallel-connected VSC HVDC connections," IET Generation, Transmission \& Distribution, vol. 10, no. 6, Apr. 2016, pp. 1336-1344.

Digital Object Identifier: $\underline{10.1049 / \text { iet-gtd.2015.0876 }}$

URL (IET Digital Library):

https://digital-library.theiet.org/content/journals/10.1049/iet-gtd.2015.0876

URL (IEEE Xplore Digital Library):

https://ieeexplore.ieee.org/document/7467049

(C) 2016 IET. This paper is a postprint of a paper submitted to and accepted for publication in IET Generation, Transmission \& Distribution and is subject to Institution of Engineering and Technology Copyright. The copy of record is available at IET Digital Library. 


\title{
Analysis of control interactions in multi-infeed VSC HVDC connections
}

\author{
Alejandro Bayo-Salas ${ }^{1,2} *$, Jef Beerten ${ }^{1}$, Johan Rimez ${ }^{2}$, Dirk Van Hertem ${ }^{1}$ \\ ${ }^{1}$ University of Leuven, Esat/Electa, Kasteelpark Arenberg 10, 3001 Heverlee, Belgium \\ ${ }^{2}$ ELIA System Operator, Blvd. de l'Empereur 20, 1000 Brussels, Belgium \\ *alejandro.bayosalas@elia.be
}

\begin{abstract}
:
The number of voltage source converters connected to the power system is increasing. This has led to concert about the impact of control interactions between converters and their influence on the system stability. Recent grid codes only regard such interactions as low frequency disturbances between the converters and hence neglect network dynamics. This paper studies the control interactions and interferences in a multi-infeed system with two converters. The phenomena is studied in the frequency domain by using the admittance-based stability assessment. Harmonic stability under different network characteristics is addressed. The analysis shows that resonances in the system can lead to instabilities when appearing within the control bandwidth, thereby implying the necessity to also consider higher frequency dynamics.
\end{abstract}

\section{Introduction}

Current incentives in the energy sector promote the installation of offshore wind farms in the North Sea. At the same time, grid planning and market integration are driving the need for more interconnection between countries. High Voltage Direct Current (HVDC) connections are often considered as the preferred solution to accommodate this increase in renewable energies and additional interconnection capacity due to their controllability and suitability for long underground and undersea connections. Nowadays, the voltage source converter (VSC) is the technology used for connecting converter-based wind generators and for transferring power through HVDC lines. In the future planning for the extension to an offshore grid, an increasing number of VSCs will be closely connected at the AC side and could mutually impact each others operation. One of the outstanding research questions is the study of possibly adverse control interactions between such converters.

When a new converter is connected to the grid, it impacts the overall AC network characteristics and the corresponding dynamics as observed at other points in the system. With the number of converters increasing, this influence will increase as well. However, multi-infeed VSC HVDC connections are not commonplace yet and current VSC control design mainly considers a simplification of the grid response at the Point of Common Coupling (PCC). Hence, unanticipated interactions with other converters, either 
because they are not planned or built or their data is not available during the process, result in stability concerns and uncertainty during the design phase.

Despite VSCs being considered as a reliable technology, suitable for the connection to any AC network [1], non-ideal characteristics of the power system have been detected to impose limitations. Particularly, the integration in weak grids is a concern. Such systems commonly represented by an equivalent perfect source behind the short-circuit impedance have been observed to impose limitations on the VSC control response [2-5].

Interactions between two converters have been studied by means of steady-state gains based on the power flow jacobian matrix [6] and the Relative Gain Array [7]. These studies only consider the network dynamics up to the fundamental frequency, i.e., interactions were mainly limited to power-flow dynamics. However, in systems with controlled devices operating in a wide bandwidth of frequencies, natural resonance frequencies from the network interact with the control bandwidth of the converter. In addition, a new stability issue has emerged when fast controlled devices are connected: the harmonic stability, i.e., a small-signal voltage stability extended to a wider frequency range. These interactions might cause power quality and stability issues as well [8-10].

Harmonic stability studies are mainly performed in the frequency domain by means of the admittancebased approach $[11,12]$. This method has been used to study the interaction between a VSC and a simplified AC system [13-15]. Extending the study to systems with multiple converters, harmonic interactions have been detected to lead to stability issues in an Offshore Wind Farm (OWF) when the number of connected turbines is increased [16], between an OWF and the collector offshore converter [17], in a parallel VSC connection composed of two VSCs [18] and between the current controllers in a converter-based distribution network composed of three converters [19].

The subject of interactions between converters has recently gained a lot of interest as shown by the creation of new working groups in CIGRÉ as B4.64 on 'Impact of AC system characteristics on HVDC VSC' or B4.67 on 'Harmonic aspects of VSCs'. The influence of a new VSC on the operation of other connected converters and the electrical distance on these interactions is still not fully understood. However, the electrical proximity is very relevant as shown by the recent Grid Code on HVDC connections from ENTSO-E [20]. According to that document, TSOs shall have the right to require and to define the scope and extent of studies which demonstrate that there is no adverse interaction when several converter stations are 'within close electrical proximity'.

However, such interactions have not been studied in detail in literature so far. Furthermore, it is not clear what the necessary studies comprise of. In addition, the notion of the influence of the electrical 


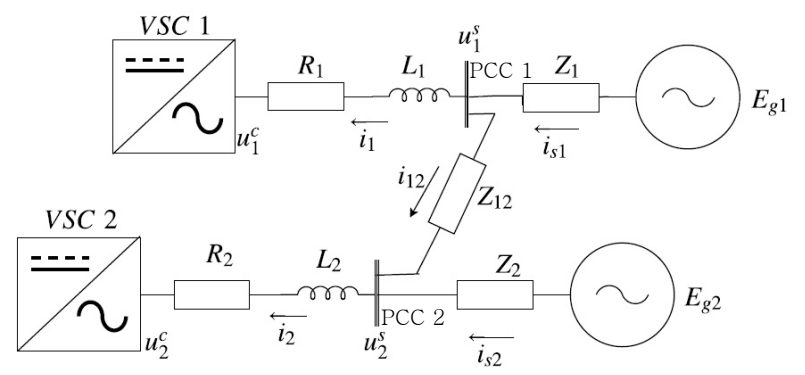

Fig. 1. System with two multi-infeed VSCs.

proximity and the AC network characteristics on the possible adverse interferences between converters remains unclear.

This paper investigates the interactions between two converters connected to different points in the AC grid, using the methodology presented in [21] and extending the analysis to a wider bandwidth of frequencies and more detailed converter models.

The problem is studied in the frequency domain and the closed-loop response of the interconnected system is evaluated. The paper assesses the emerging stability issues due to control interactions between two converters through the AC network.

\section{System modelling}

The system under study is shown in Figure 1. It consists of two converters connected to the AC system at different PCCs which are interconnected through a transmission line. The whole system is modelled by representing all elements by their reciprocal circuit equivalent models. The VSC is transformed into a current source with a parallel admittance, AC networks are converted into voltage sources behind an equivalent impedance and the transmission line is represented by the commonly used $\pi$-section representation of lines. The equivalent circuit of the studied system is depicted in Figure 2.

The problem is studied in the frequency domain. For doing so, the corresponding ordinary differential equations are linearised around an operating point. The analysis of this system is performed in the synchronous $d q$-frame rotating at the grid frequency.

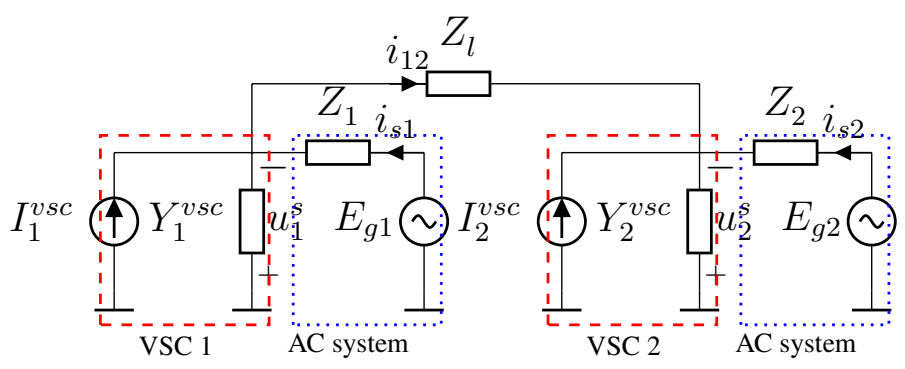

Fig. 2. Equivalent circuit of the system. 


\subsection{VSC Modelling and Control}

A way to characterize the VSC dynamics is to consider the converter to behave as a frequencydependent admittance seen from the PCC. This representation of the VSC has become widely used in stability studies $[11-13,19]$. This approach has also been used for evaluating sub-synchronous interactions between a VSC and a nearby synchronous generator [14], for interferences between the DC voltage control and the DC link [22] and the impact between the VSC and the AC network in the positive and negative sequences $[13,23]$.

Each converter is represented by its Norton equivalent circuit as a current-controlled source with a parallel admittance $Y_{v s c}$ as sketched in Figure 2. The injected controlled current to the respective PCC is a function of the external set-points and the PCC voltage $u^{s}$, regarded as a disturbance term,

$$
i_{v s c}=G(s) \cdot\left[P / Q^{r e f}\right]+Y_{v s c}(s) u^{s}
$$

where $G(s)$ is the outer loop TF and $P / Q^{r e f}$ represents the external set-point.

$Y_{v s c}$ is a transfer function from the respective input PCC voltage $u^{s}$ to the output current $i$. Since the equations and control are aligned with the synchronous $d q$ frame, the admittance $Y_{v s c}$ is a matrix of transfer functions composed by the diagonal elements related to $d$ and $q$ components and the cross-couplings in the off-diagonal terms as,

$$
\left(\begin{array}{c}
\Delta i_{d} \\
\Delta i_{q}
\end{array}\right)=\left(\begin{array}{cc}
Y_{v s c}^{d d} & Y_{v s c}^{d q} \\
Y_{v s c}^{q d} & Y_{v s c}^{q q}
\end{array}\right)\left(\begin{array}{c}
\Delta u_{d}^{s} \\
\Delta u_{q}^{s}
\end{array}\right)
$$

The frequency-dependent response of one converter can be derived either by deriving analytically the transfer function from all the equations relating the input and output or alternatively by obtaining the TF from the state space matrices. The latter is used in the paper since modifications and additions in the control can be more easily implemented.

The control is implemented in the $d q$ reference frame synchronised to the fundamental frequency. The basic control principle for the VSC is based on a two-level cascaded control, in which active and reactive power are controlled independently. Since the study is only focusing on the dynamics in the AC system, the DC link voltage is considered constant and thus the dynamics from the DC side are not considered in this paper.

For each of the VSCs, the dynamic system equations are linearised as independent subsystems and connected according to Figure 3. 


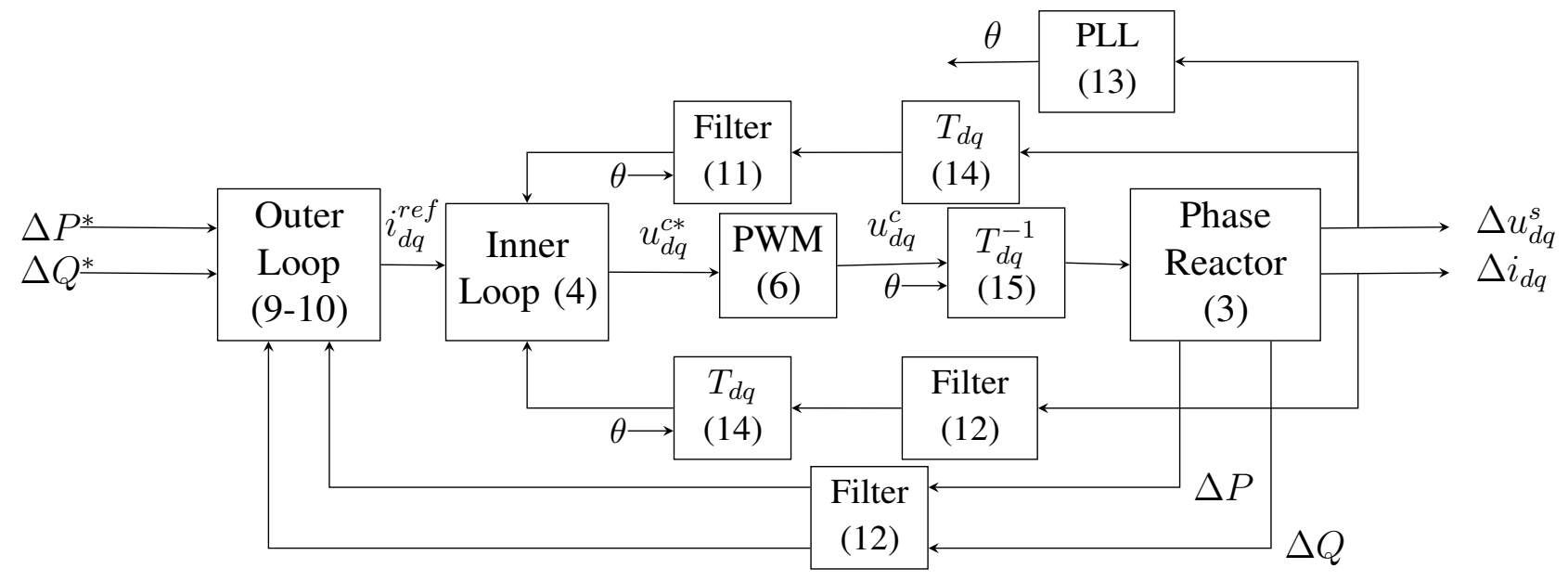

Fig. 3. Scheme of the linearised system of the VSC. The relevant system equations of each block are indicated within brackets.

Phase Reactor Equations. The series impedance dynamics in the synchronous rotatory $d q$-frame at the fundamental frequency are modelled by

$$
R\left(\begin{array}{c}
\Delta i_{d} \\
\Delta i_{q}
\end{array}\right)+L \frac{d}{d t}\left(\begin{array}{c}
\Delta i_{d} \\
\Delta i_{q}
\end{array}\right)=\left(\begin{array}{c}
\Delta u_{d}^{s} \\
\Delta u_{q}^{s}
\end{array}\right)-\left(\begin{array}{c}
\Delta u_{d}^{c} \\
\Delta u_{q}^{c}
\end{array}\right)+\left(\begin{array}{c}
-\omega L \Delta i_{q} \\
\omega L \Delta i_{d}
\end{array}\right)
$$

where $R+L s$ is the VSC series impedance, $u_{d q}^{s}$ are the voltages at the respective PCC and $u_{d q}^{c}$ the synthesized voltages at the VSC terminals of the respective converter.

Inner Control Loop Equations. The current controller provides the voltage references $u_{d q}^{c *}$ sent to the switching valves,

$$
\left(\begin{array}{c}
\Delta u_{d}^{c *} \\
\Delta u_{q}^{c *}
\end{array}\right)=-\left(\begin{array}{l}
G_{c c}^{d}(s) \\
G_{c c}^{q}(s)
\end{array}\right)\left(\begin{array}{c}
\Delta i_{d}^{r e f}-\Delta i_{d} \\
\Delta i_{q}^{r e f}-\Delta i_{q}
\end{array}\right)+w L\left(\begin{array}{c}
-\Delta i_{q} \\
\Delta i_{d}
\end{array}\right)+\left(\begin{array}{c}
\Delta \hat{u}_{d}^{s} \\
\Delta \hat{u}_{q}^{s}
\end{array}\right)
$$

where $\hat{u}_{d q}^{s}$ are the measured -and filtered-grid voltages and $G_{c c}$ represents the PI current controller,

$$
G_{c c}(s)=K_{p}^{c c}+\frac{K_{i}^{c c}}{s}
$$

PWM Switching delay. $\quad D_{p w m}$, shown as PWM in Figure 3, from $u_{d q}^{c *}$ to $u_{d q}^{c}$ is modelled as a first-order Padé approximation.

$$
D_{p w m}(s)=\frac{1}{t_{p w m} s+1}
$$


Outer Control Loop Equations. The $d q$ reference currents in Equation 4 are computed by the outer loops which track the active and reactive power set-point, $P^{r e f}$ and $Q^{r e f}$, respectively. The dynamic model linearised around the operating point $\left(i_{d 0}=\frac{P_{0}}{u_{d 0}^{s}}, i_{q 0}=-\frac{Q_{0}}{u_{d 0}^{s}}\right)$ is,

$$
\begin{aligned}
& \Delta i_{d}^{r e f}=G_{p}\left(P^{r e f}-\Delta P\right) \\
& \Delta i_{q}^{r e f}=G_{q}\left(Q^{r e f}-\Delta Q\right)
\end{aligned}
$$

where $G_{p}$ and $G_{q}$ are the PI controls of the active and reactive power controller respectively and $\Delta P$ and $\Delta Q$ are calculated by,

$$
\begin{gathered}
\Delta P=u_{d 0}^{s} \Delta i_{d}+i_{d 0} \Delta u_{d}^{s}+i_{q 0} \Delta u_{q}^{s} \\
\Delta Q=-i_{q 0} \Delta u_{d}^{s}-u_{d 0}^{s} \Delta i_{q}+i_{d 0} \Delta u_{q}^{s}
\end{gathered}
$$

Digital filters. Noise and harmonics in the measured signals are attenuated through a first-order low-pass filter for the feed-forward voltage and through a second-order filter for power and current measurements according to the following equations,

$$
\begin{gathered}
F_{f f}=\frac{\alpha_{f f}}{s+\alpha_{f f}} \\
F_{2 n d}=\frac{\omega_{0}^{2}}{s^{2}+2 \omega_{0} \xi s+\omega_{0}^{2}}
\end{gathered}
$$

Phase Locked Loop equations. The PLL is used to estimate the angle which orientates $d q$ components in the control to the grid angle of the network. It is linearised according to [11] where the small signal deviation of the estimated grid angle is represented by the transfer function,

$$
\Delta \theta=-\frac{K_{p-P L L} s+K_{i-P L L}}{s^{2}+u_{d 0}^{s} K_{p-P L L} s+u_{d 0}^{s} K_{i-P L L}} \Delta u_{q}^{s}
$$

Park Transformation equations. The $d q$ reference frame synchronised to the estimated frequency in the converter is rotated in relation to the common $D Q$ frame synchronised to the fundamental depending the small angle deviation introduced by the PLL. This relation is given by the transform $\left[T_{d q}\right]$ and the inverse 
Fig. 4. $\pi$ section transmission line representation

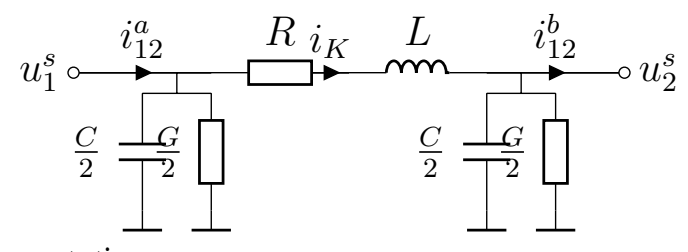

$\left[T_{d q}\right]^{-1}$ which are linearised around the operating point $\left(\Delta \theta_{0}, x_{d 0}, x_{q 0}\right)$ as,

$$
\begin{gathered}
{\left[T_{d q}\right]=\left(\begin{array}{ccc}
\cos \left(\theta_{0}\right) & \sin \left(\theta_{0}\right) & \cos \left(\theta_{0}\right) x_{d 0}-\sin \left(\theta_{0}\right) x_{q 0} \\
-\sin \left(\theta_{0}\right) & \cos \left(\theta_{0}\right) & -\cos \left(\theta_{0}\right) x_{d 0}-\sin \left(\theta_{0}\right) x_{q 0}
\end{array}\right)} \\
{\left[T_{d q}\right]^{-1}=\left(\begin{array}{ccc}
\cos \left(\theta_{0}\right) & -\sin \left(\theta_{0}\right) & -\sin \left(\theta_{0}\right) x_{d 0}-\cos \left(\theta_{0}\right) x_{q 0} \\
\sin \left(\theta_{0}\right) & \cos \left(\theta_{0}\right) & \cos \left(\theta_{0}\right) x_{d 0}-\sin \left(\theta_{0}\right) x_{q 0}
\end{array}\right)}
\end{gathered}
$$

\subsection{Transmission line}

In this paper, transients caused by the electronic components switching are not considered and thus neither the resulting harmonics at those frequencies. The transmission line parameters used in Table 1 with $\gamma l<1$-where $\gamma$ is the propagation constant- allow to approximate the model to an equivalent $\pi$ section with lumped parameters with enough accuracy in the bandwidth of interest.

The behaviour of the transmission line composed by one $\pi$ section in Figure 4 is represented by the equations

$$
\begin{aligned}
\frac{d}{d t} u_{1}^{s} & =\frac{2}{C} i_{12}^{a}-\frac{2}{C} i_{K}-\frac{G}{C} u_{1}^{s} \\
\frac{d}{d t} i_{K} & =\frac{1}{L} u_{1}^{s}-\frac{1}{L} u_{2}^{s}-\frac{R}{L} i_{K} \\
\frac{d}{d t} u_{2}^{s} & =\frac{2}{C} i_{K}-\frac{2}{C} i_{12}^{b}-\frac{G}{C} u_{2}^{s}
\end{aligned}
$$

Table 1 Parameters of the transmission line $\pi$ model.

\begin{tabular}{|l|l|}
\hline$\pi$ parameters & Values \\
\hline Resistance & $40 \mathrm{~m} \Omega / \mathrm{km}$ \\
Inductance & $1.3 \mathrm{mH} / \mathrm{km}$ \\
Capacitance & $0.15 \mu \mathrm{S} / \mathrm{km}$ \\
\hline
\end{tabular}




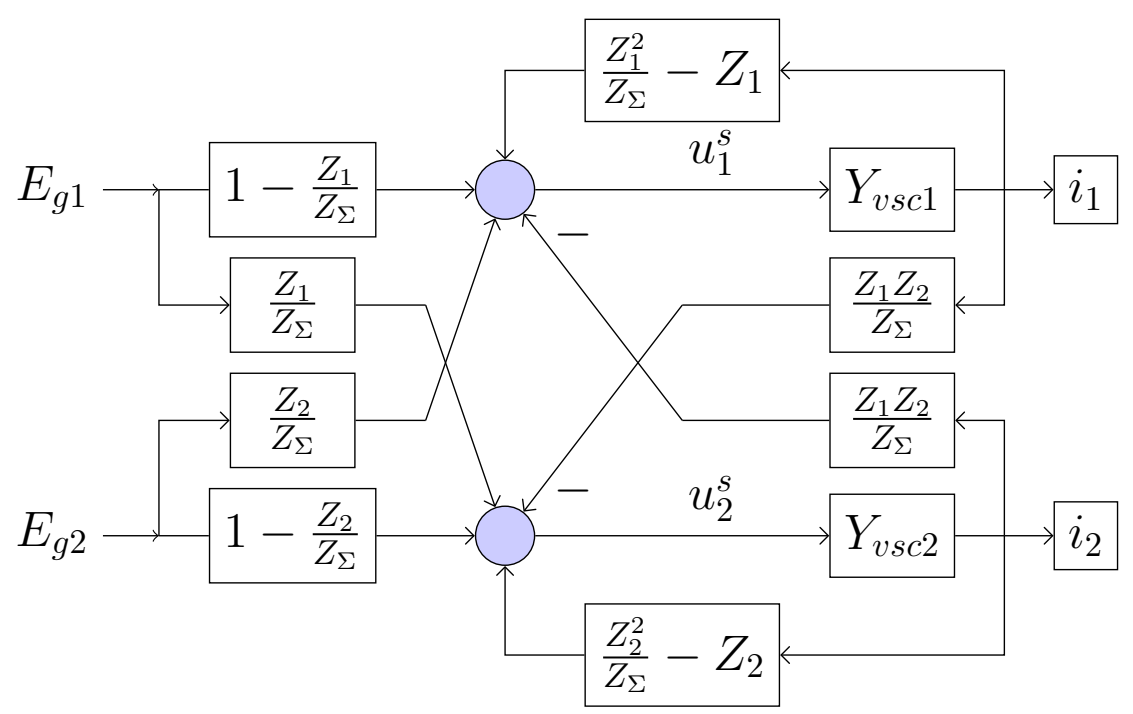

Fig. 5. MIMO system of interacting multi infeed-connected converters.

\subsection{AC network}

One of the most basic and simple ways to model the AC network is by means of representing it as a voltage source behind an impedance. The series RL represents the short-circuit impedance at the node as an approximation of the system strength at the PCC. It is defined by the quotient $\frac{|V|^{2}}{S_{s c}}$, where $S_{s c}$ is the short-circuit power. The equivalent resistance $R_{a c}$ and inductance $L_{a c}$ are determined by the $X / R$ ratio.

Since the analysis is performed in the $d q$ frame, all the network side dynamics are converted into the rotatory frame being the resulting matrix equal to,

$$
Z_{k}(s)=\left(\begin{array}{cc}
R_{a c}+s \cdot L_{a c} & -\omega L_{a c} \\
\omega L_{a c} & R_{a c}+s \cdot L_{a c}
\end{array}\right) \quad \text { for } k=1,2
$$

\section{Interactions between multi-infeed voltage source converters}

In this section, the methodology for studying the interactions is developed and explained. To that end, a Multi-Input Multi-Output (MIMO) system composed of two Single-Input Single-Output (SISO) systems is formulated. Each of the SISO systems corresponds to one VSC. Their couplings are a function of the network impedances of the circuit. The complete system is sketched in Figure 5.

The SISO system explained in $[11,12]$ is extended to the system depicted in Figure 2 with two converters. Hence the current injected at the PCC from the VSC is,

$$
i_{k}=I_{v s c, k}+Y_{v s c, k} u_{k}^{s} \quad k=1,2
$$


The PCC voltage is defined as,

$$
u_{k}^{s}=E_{g k}-Z_{k} i_{s k}
$$

where $i_{s k}$ is the current injected to the AC grid equal to $i_{s k}=i_{k}+i_{12}$ and the current through the line $i_{12}$ is derived from,

$$
Z_{l} i_{12}=\left(u_{1}^{s}-u_{2}^{s}\right)
$$

Substituting (21) into (22), the PCC voltages are obtained as function of the Thevenin voltages and the output currents as,

$$
\begin{aligned}
& u_{1}^{s}=\left(1-\frac{Z_{1}}{Z_{\Sigma}}\right) E_{g 1}+\left(\frac{Z_{1}^{2}}{Z_{\Sigma}}-Z_{1}\right) i_{1}+\frac{Z_{1}}{Z_{\Sigma}} E_{g 2}-\frac{Z_{1} Z_{2}}{Z_{\Sigma}} i_{2} \\
& u_{2}^{s}=\left(1-\frac{Z_{2}}{Z_{\Sigma}}\right) E_{g 2}+\left(\frac{Z_{2}^{2}}{Z_{\Sigma}}-Z_{2}\right) i_{2}+\frac{Z_{2}}{Z_{\Sigma}} E_{g 1}-\frac{Z_{1} Z_{2}}{Z_{\Sigma}} i_{1}
\end{aligned}
$$

where $Z_{\Sigma}=Z_{1}+Z_{2}+Z_{l}$.

From Equations (23) and (24), one can observe that PCC voltages, $u_{k}^{s}$, are influenced by both AC systems, the feedback from the current injected by the VSC connected to that PCC and also the controlled current from the other VSC, which is in turn also influenced by the current from the first converter.

The complete MIMO system is represented by Figure 5 where the transfer functions relating the different variables are obtained from Equations (23) to (24).

The closed-loop stability from the stiff grid voltages to the controlled currents is studied from the system defined by,

$$
\begin{aligned}
& i_{1}=Y_{11}^{c l} E_{g 1}+Y_{12}^{c l} E_{g 2} \\
& i_{2}=Y_{21}^{c l} E_{g 1}+Y_{22}^{c l} E_{g 2}
\end{aligned}
$$

These transfer functions relating inputs and outputs in Equation (25) are obtained by substituting Equation (23) and (24) into (20) and isolating the current cross-couplings. The closed-loop admittance of one converter is equal to,

$$
Y_{v s c 1}^{c l}=\frac{Y_{v s c, 1}\left(1-\frac{Z_{1} Z_{2} Y_{v s c, 2}}{Z_{\Sigma}\left(1-Y_{v s c, 2}\left(\frac{Z_{2}^{2}}{Z_{\Sigma}}-Z_{2}\right)\right)}\right)}{1-Y_{v s c, 1}\left(\frac{Z_{1}^{2}}{Z_{\Sigma}}-Z_{1}+\frac{Z_{1}^{2} Z_{2}^{2} Y_{v s c, 2}}{Z_{\Sigma}^{2}\left(1-Y_{v s c, 2}\left(\frac{Z_{2}^{2}}{Z_{\Sigma}}-Z_{2}\right)\right)}\right)}
$$

Interactions are thus reflected by means of the closed-loop admittances of the system $Y_{i j}^{c l}$. Each of these 
transfer functions has been derived by considering all the possible paths and hence are influenced by all the elements which compose the network.

\section{Dynamic Analysis}

The stability of a system can be studied by one of the properties that dynamic systems exhibit: its passivity. In short, a linear system is passive if it dissipates energy and oscillations are attenuated as an analogy to the behaviour of passive electrical circuits. When a dynamic system is passive along its whole range of operation, the system is stable in the sense of the Lyapunov criteria [24], i.e., the system will have a bounded energy output for a given bounded energy input.

Particularly, electrical components are passive when their frequency-domain interpretation exhibits a phase within $-90^{\circ} \leq \varphi \leq 90^{\circ}$ and its Nyquist diagram completely lies on the Right Half Plane (RHP) and the real part of the transfer function is positive. On the other hand, regions where $\Re[Y(\jmath \omega)]<0$ indicate frequencies at which the system adds negative damping to the network and hence amplifies oscillations at those frequencies.

Ideally, beyond the frequency bandwidth of the current controller, the VSC dynamic response should be completely characterized by the frequency-dependent behaviour of the phase reactor, $L \cdot s+R$. However, due to the action of different digital filters and time delays, the ideal first-order dynamic response from the phase reactor slightly diverges and might create regions of negative damping. Different modifications in the VSC such as control parameters, additional filtering, installation of a coupling filter or use of other strategies will affect the frequency response of the converter and thus its interaction with the system. Studying the influence of changes in the parameters in the interactions and consideration of other control loops is out of the scope of this paper.

The dynamic response of a VSC with the parameters in Table 2 under different operating points is studied. For this, the VSC admittance is plotted in the RX plane and the negatively damped regions are checked.

Figures 7 and 8 show the R-X plot of the $d d$ and $q q$ components of the VSC admittance under variation of the active power operating point between $-1 \mathrm{GW}$ and $1 \mathrm{GW}$ and the reactive power between $-0.3 \mathrm{GVAr}$ and $0.3 \mathrm{GVAr}$ respectively. Two non-passive regions, where $\Re[Y(\jmath \omega)]<0$, are observed. The first one is located in the low frequency range below $3 \mathrm{~Hz}$. The second occurs in the region between $290-500 \mathrm{~Hz}$, see also Figure 7-c. This characteristic appears in all the studied components. A region of these characteristics does not pose a threat to stability as long as the interacting network dissipates energy at those frequencies.

In the paper, only diagonal terms of the matrix in Equation 2 are addressed because cross-coupling terms 


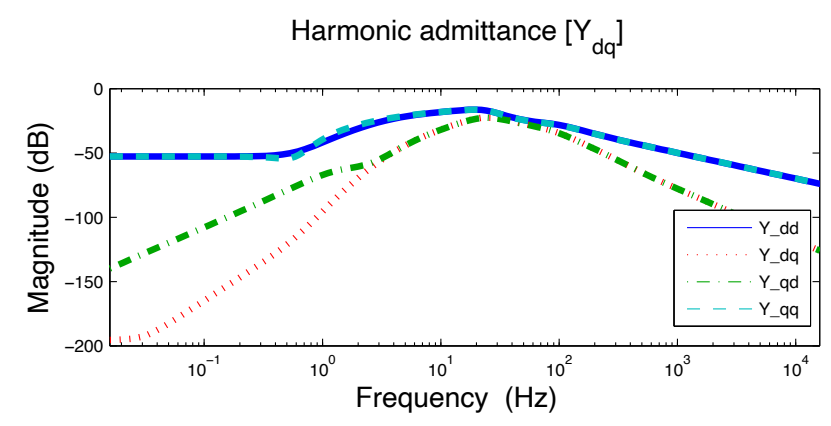

Fig. 6. Magnitudes of diagonal and cross-coupling components of the VSC input-admittance.

have a lower magnitude than diagonal terms. This is observed in Figure 6 where $d q$ and $q d$ terms are negligible in the low and high bandwidth of frequencies.

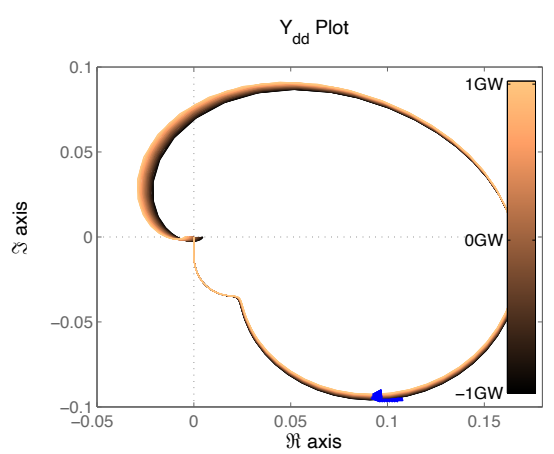

a)

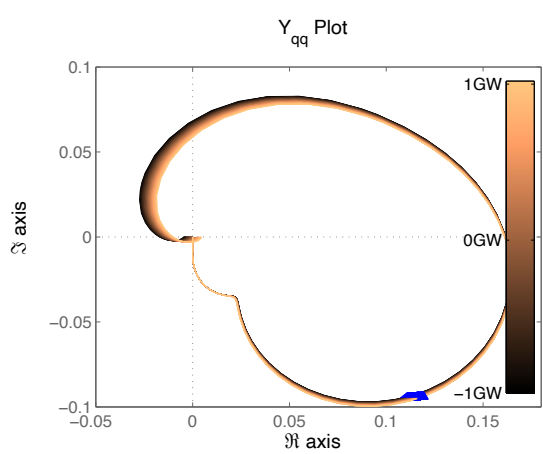

b)

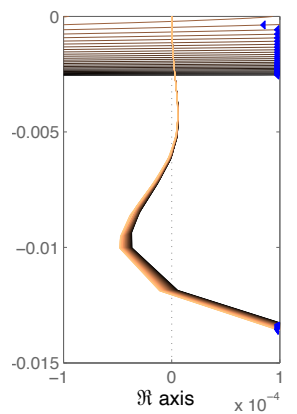

c)

Fig. 7. $\Re$ - $\Im$ plot of the VSC admittance under $P^{*}$ variation.

a $d d$ component

b $q q$ component

c Zoom in the region between $250 \mathrm{~Hz}$ to $\infty$

Table 2 VSC parameters

\begin{tabular}{|l|c|}
\hline VSC parameters & Values \\
\hline AC Voltage $V$ & $380 \mathrm{kV}$ \\
Base Power $S$ & $1.1 \mathrm{GVA}$ \\
Series Impedance & $R=0.525 \Omega \quad L=50 \mathrm{mH}$ \\
Current controller & $\frac{6.25 s+65.6}{s}$ \\
Active and reactive controller & $\frac{1 s+10}{s}$ \\
PLL & $\frac{0.5 \mathrm{~s}+50}{s}$ \\
Feed-forward filter & $\alpha_{f f}=15 \mathrm{~Hz}$ \\
Power meas. filter & $\omega=150 \mathrm{~Hz} \quad \xi=0.707$ \\
Current filter & $\omega=400 \mathrm{~Hz} \quad \xi=0.7$ \\
PWM time delay & $t_{p w m}=0.5 \mathrm{~ms}$ \\
\hline
\end{tabular}




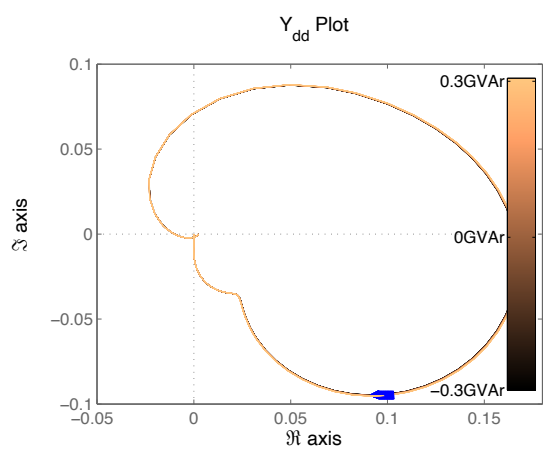

a)

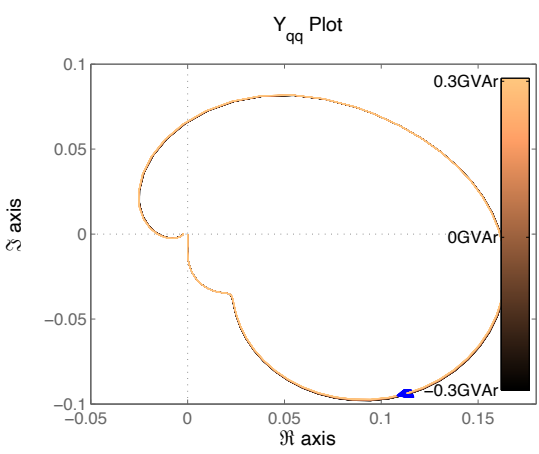

b)

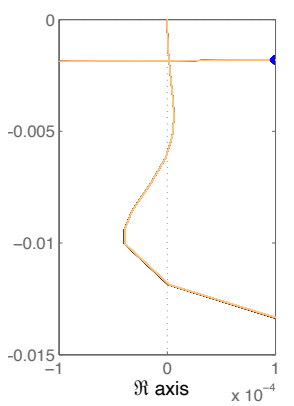

c)

Fig. 8. $\Re-\Im$ plot of the VSC admittance under $Q^{*}$ variation.

a $d d$ component

b $q q$ component

c Zoom in the region between $250 \mathrm{~Hz}$ to $\infty$

\subsection{Dynamic interactions in a multi-infeed system}

This section evaluates the dynamic response of one VSC under variations of network parameters such as the transmission line length and system strength parameters. To do so, the closed-loop transfer function of one converter considering interactions with the network and the second VSC is obtained as explained in section 3.

When a converter is interconnected and interacts with the surrounding system, new resonances and oscillatory modes appear. Resonances occur when there is a phase difference of $180^{\circ}$ at the intersection point between the two interacting system impedances. This is sketched in Figure 9 where three admittances are compared: the isolated VSC admittance modelled with the parameters in Table 2, the transfer function of the surrounding network -composed of the transmission line, short circuit impedances and the second converter-, and the closed-loop admittance, $Y_{v s c 1}^{c l}$.

Stability can be studied by common frequency domain techniques in feedback systems such as the Nyquist criterion or the Bode of the closed-loop system. As such, instabilities of the interconnected network can be detected by encirclements to the critical point $(-1+\jmath 0)$ by the Nyquist contour of the denominator in Equation 26 or when the closed-loop admittance in Equation 26 has a phase shift equal to $-180^{\circ}$ with 0 $\mathrm{dB}$ magnitude in the Bode diagram. Other important measures are the relative stability indicators such as the gain and phase margin [25]. These measures give and idea of how close the system is to be unstable under uncertainties. 

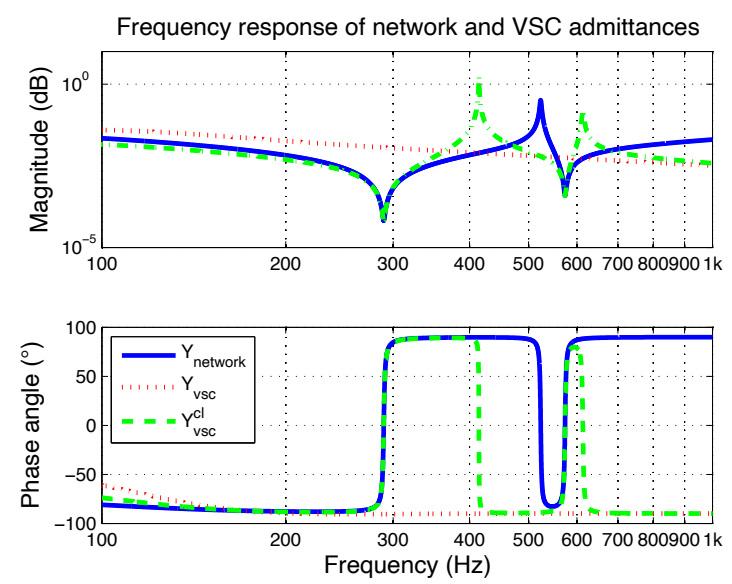

Fig. 9. Frequency response of the network admittance seen at the PCC (solid line), the VSC input-admittance (dotted line), and the closed loop VSC admittance (dashed line). Resonances occur at 410 and $570 \mathrm{~Hz}$.
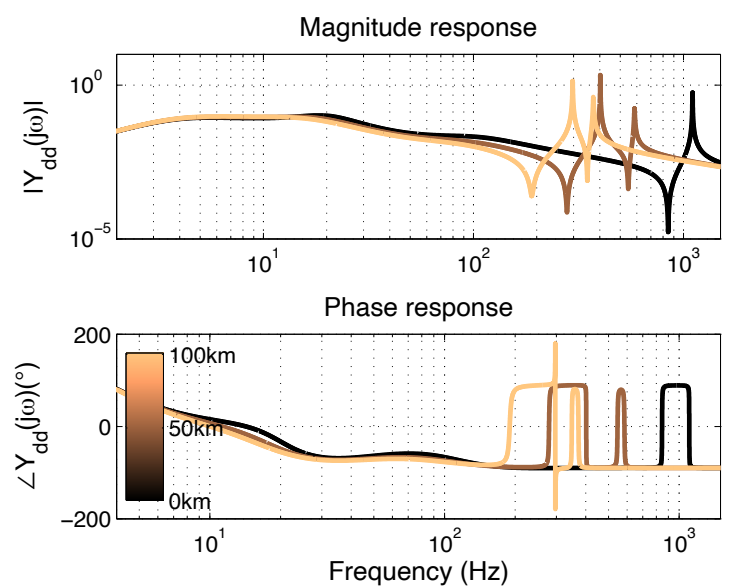

Fig. 10. Magnitude and phase of $Y_{v s c}^{c l}$ for $S C R=2, X / R=10$ under variation of the transmission line length.

\subsection{Transmission line length}

The impact of the transmission line length on the interactions is studied. Figure 10 shows the dynamic response of the interconnected system for different lengths of the line. The analysis indicates an instability when a low-damped resonant point of the interconnected system coincides with the non-passive region of the VSC. In this specific case, resonances shift to lower frequencies and closer to the control bandwidth when the transmission line length is increased. This instability is observed when the phase intersects the $-180^{\circ}$ angle. In the specific test case, this occurs when the resonant point reaches $385 \mathrm{~Hz}$.

The instability is also observed in the Nyquist diagram of the loop gain transfer function in Figure 11 when the curve encircles the critical point $-1+\jmath 0$ for a determined line length. Moreover, the proximity of the gain crossover points when the line is increased -marked in the figure by a blue dashed circle- indicates the closeness of one oscillatory mode to be unstable under changes in the network.

Finally, the influence on the instability of the second converter is observed in Figure 12. In the plot, 

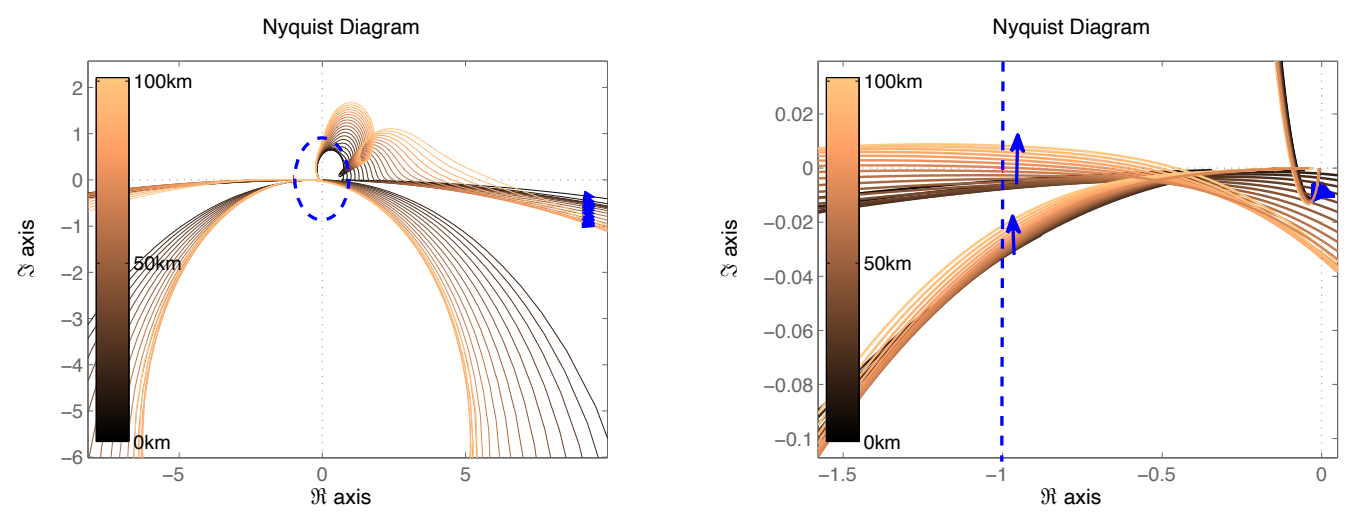

Fig. 11. Nyquist diagram and zoom around $-1+\jmath 0$ of the open-loop denominator for $S C R=2, X / R=10$ under variation of the transmission line length.
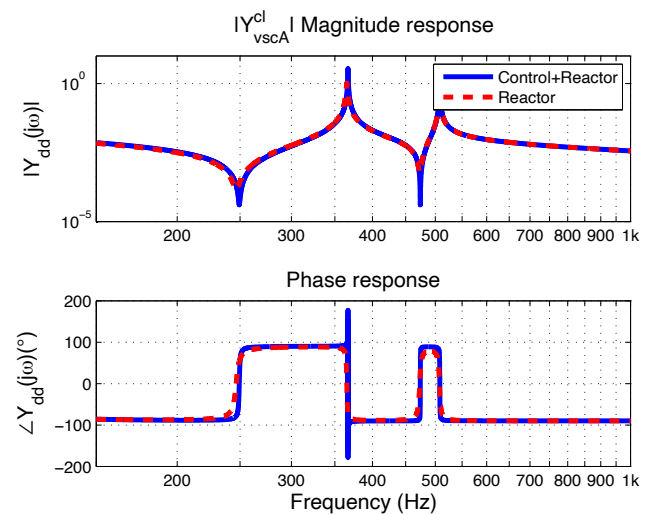

Fig. 12. Magnitude and phase of $Y_{v s c 1}^{c l}$ when the second converter includes the control in the model in solid and is only modelled by it phase reactor in dashed.

two $Y_{v s c 1}^{c l}$ closed-loop responses are compared; one by including all control dynamics in the second converter and the other by reducing the second VSC to the phase reactor dynamics. Both curves show the same resonant points, where the dominant mode is located at $365 \mathrm{~Hz}$. However, when the second VSC is only modelled by the series impedance, the unstable point is not detected and the phase is bounded within the passive region between $-90^{\circ} \leq \varphi \leq 90^{\circ}$. The model reduction can provide a first indication of the existing oscillatory modes in the system. However, neglecting the control dynamics from the other VSC leads to a wrong assessment of the system instability if some conditions are met, such as resonances within the control bandwidth or negative-admittance regions.

\subsection{AC network parameters}

Apart from the transmission line, variations in the network strength and X/R ratio can destabilize an initially stable system. Both parameters have an influence on the resonant points and damping -or energy dissipation- in the system. The influence of the network parameters on the control interactions is addressed 

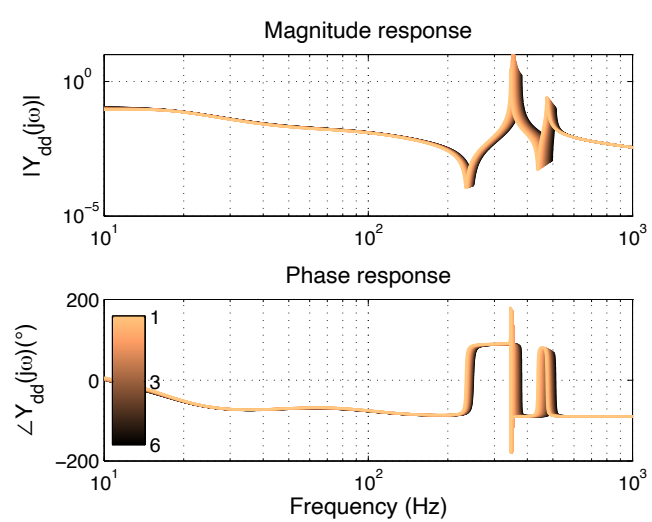

Fig. 13. Magnitude and phase of $Y_{v s c A}^{c l}$ for line length $=75 \mathrm{~km}, X / R=10$ under variation of the SCR.
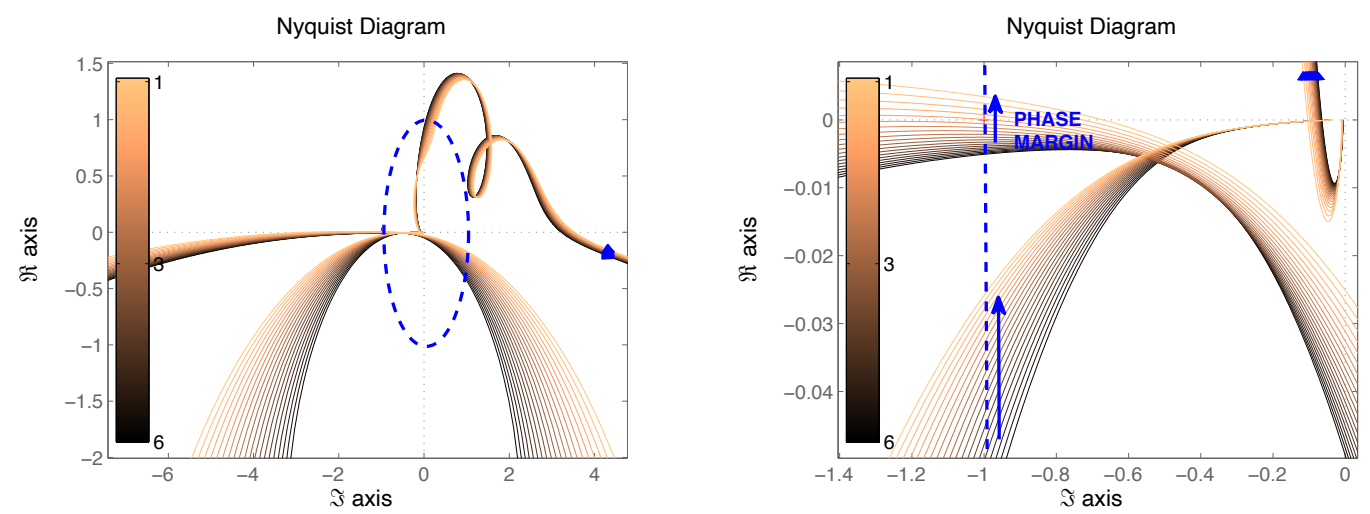

Fig. 14. Nyquist diagram and zoom around $-1+\jmath 0$ of the open-loop denominator for line length $=75 \mathrm{~km}, X / R=10 \mathrm{under}$ variation of the SCR.

by varying the short-circuit impedance magnitude the $\tan ^{-1}(X / R)$ angle .

Figures 13 and 14 show the impact of the SCR on the interactions. Only the SCR from the network to which the second VSC is connected is modified $-Z_{2}$ in Figure 1-. Decreasing the SCR causes a shift of the oscillatory modes towards the control bandwidth. The shift of the resonance frequencies is shown in the Bode diagram in Figure 13. Instabilities appear when the SCR is decreased and reaches a value of 2.2 for this specific study case.

The decrease in the SCR also makes the system more prone to be unstable. The deterioration in the relative stability is clear from the phase margin reduction indicated by the blue circle in the Nyquist diagram in Figure 14. The reduction in the phase margin will result in oscillatory modes with reduced damping.

Regarding the $\mathrm{X} / \mathrm{R}$ ratio, systems with low $\mathrm{X} / \mathrm{R}$ ratio will increase the damping at the resonances and hence they will not be as prone to instabilities as in highly inductive networks. This response is observed in Figures 15 and 16. In the figures, the instability disappears when the $\mathrm{X} / \mathrm{R}$ ratio is decreased. The phase margin analogously increases for a reduction in the $\mathrm{X} / \mathrm{R}$ ratio. This results in a transient performance and 
relative stability improvement.
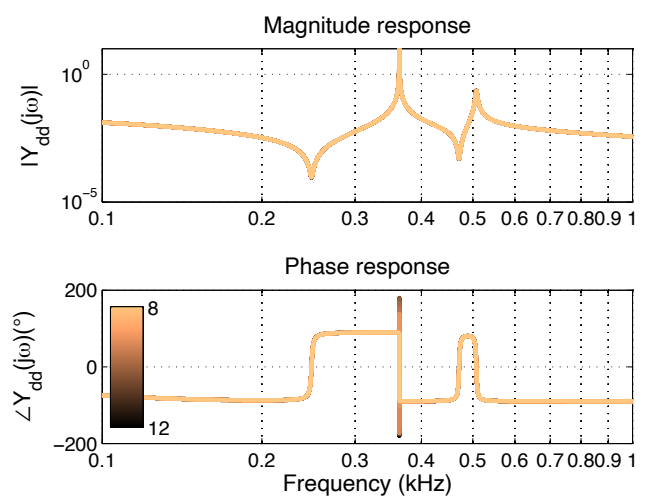

Fig. 15. Magnitude and phase of $Y_{v s c 1}^{c l}$ for line length $=65 \mathrm{~km}, S C R=2$ under variation of the XR ratio.

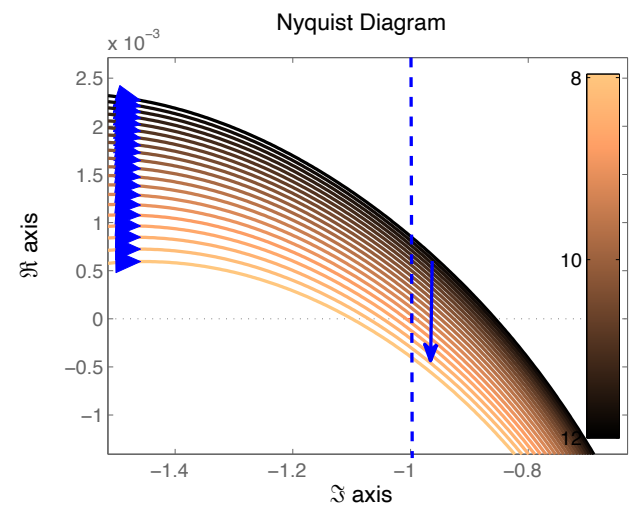

Fig. 16. Nyquist diagram of the open-loop denominator for line length $=65 \mathrm{~km}, S C R=2$ under variation of the XR ratio.

\section{Time-domain validation}

Instabilities evaluated with the impedance-based approach are validated by means of EMT simulations. The closed-loop stability of the interconnected system is investigated for two different cases. Simulations are performed in the software Simulink/SimPowerSystems. Both converters are modelled as controlled voltage sources with the parameters in Table 2.

Case I Both VSCs are connected to two separate grids represented by the voltage sources $E_{g 1}$ and $E_{g 2}$ and their respective short-circuit impedances $Z_{1}$ and $Z_{2}$. Both SCRs are equal to 3 and the X/R ratio is 10 . The two grids initially operate independently, but at $t=1 \mathrm{~s}$ the two grids are connected by switching in the transmission line. Figure 17.a shows the dynamic response of the system for two different line lengths, 55 $\mathrm{km}$ (depicted in blue) and $65 \mathrm{~km}$ (depicted in red dashed). An oscillation due to the dominant oscillatory resonant mode of the network appears. The observed oscillations are progressively damped for the case of the 55-km line, and increasing in amplitude for the $65-\mathrm{km}$ line case. The initial large transient is due to the 
connection of the line with non-initialized states. The frequencies of these oscillations are $380 \mathrm{~Hz}$ for the 65-km line and $405 \mathrm{~Hz}$ for $55 \mathrm{~km}$, as shown in the FFT of the time-domain simulation in Figure 17.b. The measured frequency of the oscillations in the FFT plot are equivalent to the determined oscillatory modes estimated by the admittance-based analysis shown in Figure 17.c. For this specific case, the dynamic analysis estimates the frequencies of oscillation and the instability for the $65-\mathrm{km}$ case (in red dashed). Therefore, the dynamic analysis performed with the admittance-based approach correctly estimated the dominant modes of oscillation and the instability in the time-domain simulation.

Case II This case verifies the stability analysis for the case in Figure 9 within Section 4.2. The second VSC is modelled as an ideal voltage source and the respective control is neglected. Both VSCs are connected to the PCC through the series reactor. Figure 18.a shows the time-domain response. Initially the transmission line is disconnected. At $\mathrm{t}=1 \mathrm{~s}$, the line with a length of $70 \mathrm{~km}$ is connected. The oscillation due to the resonance appears and is attenuated. At $\mathrm{t}=1.3 \mathrm{~s}$, the ideal voltage source is changed to the controlled VSC. As a consequence, an increasing oscillation at the same frequency appears and the system becomes unstable. Hence, when the control is considered, resonant oscillatory modes from the network become unstable due to the negative-admittance region. The frequency of these oscillations are at $365 \mathrm{~Hz}$ as shown in the FFT of the time-domain simulation in Figure 18.b. The two curves correspond to the DFT measurement in a window between $\mathrm{t}=1.1 \div 1.2 \mathrm{~s}$ (in blue) and $\mathrm{t}=1.3 \div 1.35 \mathrm{~s}$ (in red dashed). The same result in frequencies and the estimation of instability was obtained using the admittance-based approach as shown in Figure 9. In the figure, the same network was addressed for the two cases where the second converter is ideally modelled (in dashed red) and the control dynamics are considered (in blue). The dynamic analysis correctly estimated the dominant oscillatory mode and the instability when control dynamics were considered.

\section{Conclusions}

In this paper, harmonic control interactions between nearby connected voltage source converters have been investigated. With this, the influence of changes in the interconnecting network has been addressed.

Stability issues have been encountered when new oscillatory modes appear in high frequencies close to the control bandwidth. When these systems are interconnected, poorly damped oscillatory modes appear above the fundamental frequency. These modes correspond to resonances between the network and the converter. Instabilities arise when these low-damped resonances coincide with negative-conductance regions in the connected converters. Results in the paper show that the model reduction of a converter to its phase reactor can provide a first indication of the resultant resonances. However, if high frequency modes are 


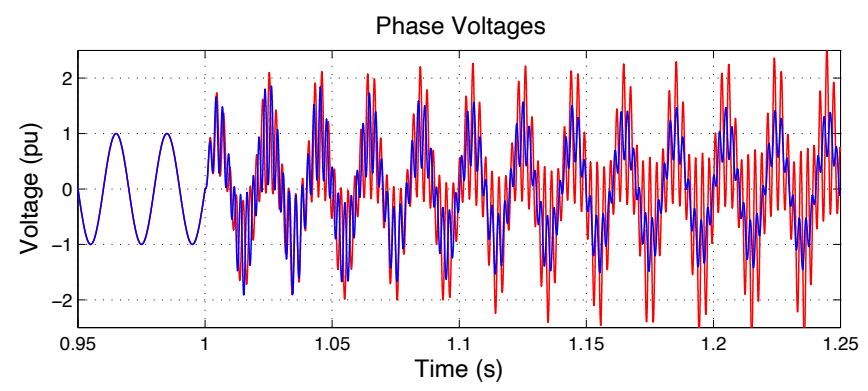

a)

Frequency spectrum of the phase voltage

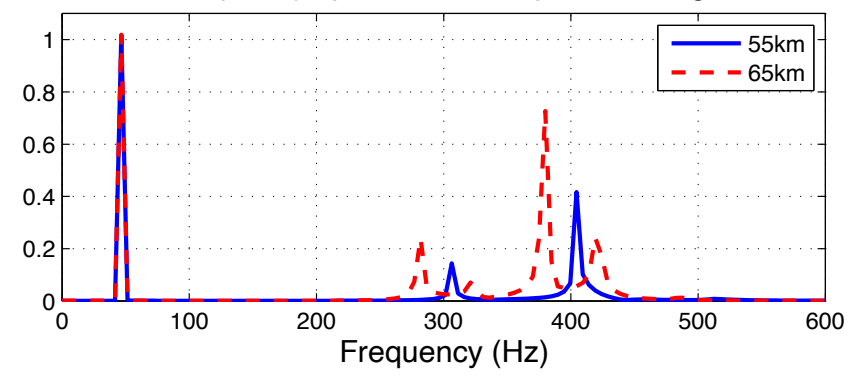

b)
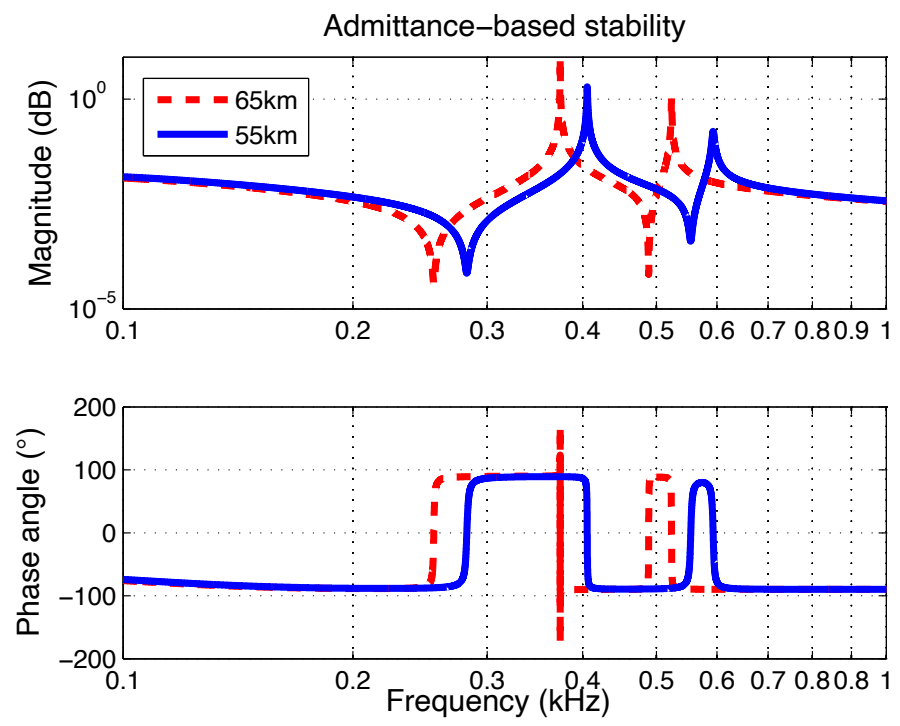

c)

Fig. 17. EMT simulation for the Case I with $S C R s=3$ and $X / R=10$. At $t=1 \mathrm{~s}$, the transmission line is connected. $65-\mathrm{km}$ line (in red dashed) and 55-km line (in blue)

a Phase a voltage

b DFT applied in the window between $\mathrm{t}=1 \div 1.25 \mathrm{~s}$.

c Frequency response of the VSC closed-loop admittances.

located within the control bandwidth, the converter model must include the control dynamics, otherwise, it will lead to an incorrect stability assessment.

Finally the influence of the AC network characteristics on the interactions has been explored and results have been validated by means of time-domain simulations. The analysis demonstrates that the 


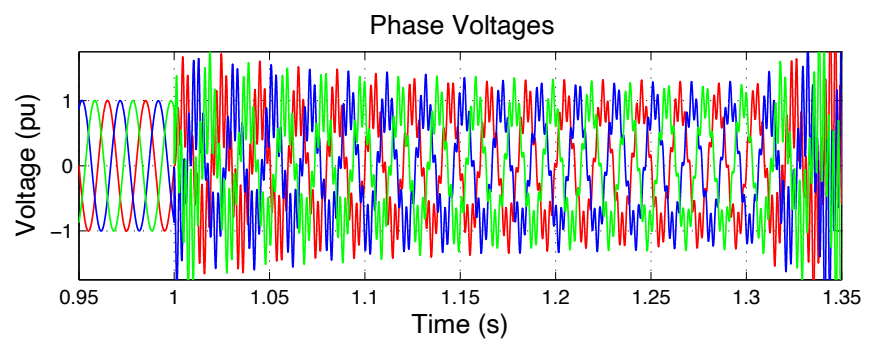

a)

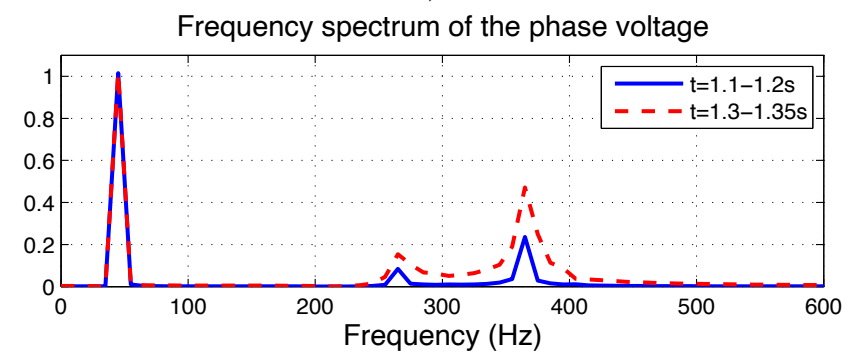

b)

Fig. 18. EMT simulation for the Case I. At $t=1$, the transmission line is connected.

a Three-phase voltages

b DFT applied in the window between $\mathrm{t}=1.1 \div 1.2 \mathrm{~s}$ (in blue) and $\mathrm{t}=1.3 \div 1.35 \mathrm{~s}$ (in red dashed).

'electrical proximity' as an interaction indicator is incomplete when the non-linearities of the network and negative damping regions of the converter are considered. Therefore assumptions about the propagation of disturbances based on the power flow phasors and the proportional increase of dissipation from the network in low frequencies are incomplete when assessing the system stability and the consideration of high frequency network modes and harmonic response from the VSC is necessary.

\section{Acknowledgements}

The research leading to these results has received funding from the People Programme (Marie Curie Actions) of the European Union's Seventh Framework Programme FP7/2007-2013/ under REA grant agreement no. 317221, project title MEDOW.

Jef Beerten is funded by a post-doctoral fellowship from the Research Foundation Flanders (FWO).

\section{References}

[1] Technichal Brochure 492, "Voltage Source Converter (VSC) HVDC for Power Transmission, Economic Aspects and Comparison with other AC and DC Technologies (WG B4)," Cigré, Tech. Rep., April 2012.

[2] J. Zhou, A. Rajapakse, and A. Gole, "VSC transmission limitations imposed by AC system strength and AC impedance characteristics," in Proc. in 10th International Conference on AC and DC Power Transmission (ACDC 2012) Birmingham, December 2012, 6 pages.

[3] L. Zhang, L. Harnefors, and H. Nee, "Power-Synchronization Control of Grid-Connected Voltage-Source 
Converters," IEEE Transactions on Power Systems, vol. 25, no. 2, pp. 809-820, May 2010.

[4] J. Zhou, H. Ding, S. Fan, Y. Zhang, and A. Gole, "Impact of Short-Circuit Ratio and Phase-Locked-Loop Parameters on the Small-Signal Behavior of a VSC-HVDC Converter," IEEE Transactions on Power Delivery, vol. 29, no. 5, pp. 2287-2296, October 2014.

[5] A. Egea-Alvarez, S. Fekriasl, F. Hassan, and O. Gomis-Bellmunt, "Advanced Vector Control for Voltage Source Converters Connected to Weak Grids," IEEE Transactions on Power Systems, vol. Early Access article, 2015, 9 pages.

[6] C.Guo, Y. Zhang, A.Gole, and C.Zhao, "Analysis of Dual-Infeed HVDC with LCC and VSC-HVDC," IEEE Transactions on Power Delivery, vol. 27, no. 3, pp. 1529-1537, July 2012.

[7] L. Shen, M. Barnes, J. V. Milanovic, K. Bell, and M. Belivanis, "Potential interactions between VSC HVDC and STATCOM," in Proc. in 18th Power Systems Computation Conference (PSCC) Warsaw, July 2014, 7 pages.

[8] P. Brogan, "The stability of multiple, high power, active front end voltage sourced converters when connected to wind farm collector system," in Proceedings in European Conference in Power Electronics and Applications, 2010, 6 pages.

[9] H. Enslin and P. J. Heskes, "Harmonic interaction between a large number of distributed power inverters and the distribution network," IEEE Transactions on Power Electronics, vol. 19, no. 6, pp. 1586-1593, November 2004.

[10] E. Mollerstedt and B. Bernhardsson, "Out of control because of harmonics-an analysis of the harmonic response of an inverter locomotive," IEEE Control Systems, vol. 20, no. 4, pp. 70-81, August 2000.

[11] L. Harnefors, M. Bongiorno, and S. Lundberg, "Input-admittance calculation and shaping for controlled voltagesource converters," IEEE Transactions on Industrial Electronics, vol. 54, no. 6, pp. 3323-3334, December 2007.

[12] J. Sun, "Impedance-based stability criterion for grid-connected inverters," IEEE Transactions on Power Electronics, vol. 26, no. 11, pp. 3075-3078, November 2011.

[13] M. Cespedes and J. Sun, "Impedance modeling and analysis of grid-connected voltage-source converters," IEEE Transactions on Power Electronics, vol. 29, no. 3, pp. 1254-1261, March 2014.

[14] K. Alawasa, Y. Abdel-Rady, and W. Xu, "Modeling, analysis and suppression of the impact of full-scale windpower converters on subsynchronous damping," IEEE Systems Journal, vol. 7, no. 4, pp. 700-712, December 2013.

[15] H. Liu and J. Sun, "Voltage Stability and Control of Offshore Wind Farms With AC Collection and HVDC Transmission," IEEE Journal of Emerging and Selected Topics in Power Electronics, vol. 2, no. 4, pp. 1181-1189, December 2014.

[16] L. H. Kocewiak, J. Hjerrild, and C. L. Bak, "Wind turbine converter control interaction with complex wind farm systems," IET Renewable Power Generation, vol. 7, no. 4, pp. 380-389, July 2013.

[17] J. Glasdam and L. H. Kocewiak, "Control System interaction in the VSC-HVDC Grid Connected Offshore Wind 
Power Plant," in Cigre B4 Simposium. Across borders - HVDC Systems and Market Integration in Lund., May 2015, 8 pages.

[18] C. Wan, M. Huang, C. Tse, and X. Ruan, "Stability of interacting grid-connected power converters," Journal of Modern Power Systems and Clean Energy, Springer, vol. 1, no. 3, pp. 249-257, December 2013.

[19] X. Wang, F. Blaabjerg, and W. Wu, "Modeling and analysis of harmonic stability in an AC power-electronicsbased power system," IEEE Transactions on Power Electronics, vol. 29, no. 12, pp. 6421-6432, December 2014.

[20] ENTSO-E. Draft Network Code on HVDC Connections and DC-connected Power Park Modules (Article 27). [Online]. Available: Acces is https://www.entsoe.eu/major-projects/network-code-development/high-voltagedirect-current/Pages/default.aspx

[21] A. Bayo-Salas, J. Beerten, J. Rimez, and D. Van Hertem, "Impedance-based stability assessment of parallel VSC HVDC grid connections," in Proc. in 11th International Conference on AC and DC Power Transmission (ACDC 2015) Birmingham, February 2015, 9 pages.

[22] G. Pinares, "Analysis of the DC dynamics of VSC-HVDC systems connected to weak AC grids using a frequency domain approach," in Proc. in Power Systems Computation Conference (PSCC) Warsaw, July 2014, 7 pages.

[23] M. Cespedes and J. Sun, "Mitigation of Inverter-Grid Harmonic Resonance by Narrow-Band Damping," IEEE Journal of Emerging and Selected Topics in Power Electronics, vol. 2, no. 4, pp. 1024 - 1031, October 2014.

[24] J. C. Willems, "Dissipative dynamical systems, part i: General theory," Archive for Rational Mechanics and Analysis, vol. 45, no. 5, pp. 321-351, January 1972.

[25] K. Ogata, Modern Control Engineering. Fifth Edition., Pearson, Ed. Prentice Hall, 2009. 